山्山FANÇAISE

$>\mathrm{DE}$

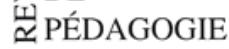

\section{Revue française de pédagogie}

Recherches en éducation

205 | 2018

Instruction(s) en famille. Explorations sociologiques d'un phénomène émergent

\title{
FOUQUET-CHAUPRADE Barbara \& SOUSSI Anne (dir.). Pratiques pédagogiques et éducation prioritaire
}

Berne : Peter Lang, 2018, 224 p.

\section{Anne Armand}

\section{OpenEdition \\ Journals}

Édition électronique

URL : https://journals.openedition.org/rfp/8760

DOI : $10.4000 /$ rfp.8760

ISSN : 2105-2913

Éditeur

ENS Éditions

Édition imprimée

Date de publication : 30 décembre 2018

Pagination : 119-122

ISBN : 979-10-362-0223-0

ISSN : 0556-7807

Référence électronique

Anne Armand, «FOUQUET-CHAUPRADE Barbara \& SOUSSI Anne (dir.). Pratiques pédagogiques et éducation prioritaire », Revue française de pédagogie [En ligne], 205 | 2018, mis en ligne le 30 décembre 2018, consulté le 15 janvier 2023. URL : http://journals.openedition.org/rfp/8760 ; DOI : https:// doi.org/10.4000/rfp.8760

Ce document a été généré automatiquement le 15 janvier 2023.

Tous droits réservés 


\section{FOUQUET-CHAUPRADE Barbara \& SOUSSI Anne (dir.). Pratiques pédagogiques et éducation prioritaire}

Berne : Peter Lang, 2018, 224 p.

\section{Anne Armand}

\section{RÉFÉRENCE}

FOUQUET-CHAUPRADE Barbara \& SOUSSI Anne (dir.). Pratiques pédagogiques et éducation prioritaire. Berne : Peter Lang, 2018, 224 p.

1 Cet ouvrage se propose de contribuer à l'objectif de « mieux comprendre ce qu'il en est des pratiques pédagogiques dans les contextes d'enseignement prioritaire ». Mais cet objectif n'est que partiellement atteint et le titre et le sommaire desservent quelque peu l'ouvrage. Il ne s'agit pas d'une revue des pratiques pédagogiques en éducation prioritaire, malgré l'effet de répétition dans le titre de chacune des trois parties, et bien que l'introduction de l'ouvrage se termine sur le souhait que le lecteur y trouve un "panorama assez large des pratiques pédagogiques en éducation prioritaire ». Le panorama proposé n'est de fait pas très large: quatre contributions se réfèrent à l'éducation prioritaire française, et deux au REP de Genève ; seules une allusion rapide à des politiques prescriptives aux États-Unis et en Angleterre et la conclusion de G. Felouzis ouvrent une perspective effectivement plus panoramique. Sur les six contributions étudiant des situations pédagogiques, la surreprésentation du primaire (cycle 2 et 3) et des apprentissages linguistiques ne permet pas non plus de témoigner largement des pratiques pédagogiques en éducation prioritaire. Le « et » du titre a donc toute son importance.

2 La lecture des neuf contributions construit deux thèses: d'une part, l'inefficacité de l'éducation prioritaire tient au déficit de prescription en matière de pratiques pédagogiques, au poids des représentations des enseignants (de leurs attentes) sur les 
apprentissages, au fait de ne pas mettre en œuvre un enseignement explicite ; d'autre part, la conclusion du rapport du CNESCO (2016) selon laquelle existeraient des différences de qualité d'enseignement en et hors éducation prioritaire parce que, s'adaptant à leurs élèves, les enseignants reverraient à la baisse leurs exigences et les curriculum, avec des conséquences sur les apprentissages des élèves, n'est pas confirmée par les enquêtes dont l'ouvrage se fait l'écho; ni en France ni à Genève la qualité de l'enseignement serait moindre en éducation prioritaire que hors éducation prioritaire.

\section{Trois explications de « l'échec » des politiques d'éducation prioritaire sont avancées}

Déficit de prescription pédagogique: les textes réglementant la politique d'éducation prioritaire, jusqu'à la publication, en France, du Référentiel de l'éducation prioritaire en 2014, n'ont jamais été prescriptifs en matière de pédagogie ; ils ont défini sans réel fondement des principes de réussite (innover, travailler en groupe, réduire le nombre d'élèves par classe) sans pour autant outiller les enseignants, ni leur proposer des ressources théoriques et pratiques aux résultats avérés, au nom de la confiance faite au terrain : forts de leur bonne volonté, les enseignants sont censés trouver les meilleures solutions (Rochex; Fouquet-Chauprade \& Dutrévis). La pédagogie n'a pas été choisie comme levier décisif pour faire progresser les apprentissages. Or les enseignants n'ont pas nécessairement conscience des pédagogies qu'ils mettent en œuvre, ni que les différentes pratiques pédagogiques auxquelles sont confrontés les élèves peuvent être en contradiction les unes avec les autres. Les stratégies pédagogiques mises en œuvre peuvent demeurer «invisibles» ou ne pas avoir les effets démocratisants attendus. D'où l'importance du cadrage du dispositif, de la formation des acteurs, de la mise à disposition d'outils didactiques dont la systématisation des entrainements et de l'enseignement explicite est illustrée dans les contributions de Bressoux et Bianco (PARLER, apprentissage phonologique et code alphabétique, cycle 2) et de Chesnais (différenciation en mathématiques en sixième).

4 Représentations: une autre explication est celle des attentes des acteurs liées à leurs représentations (Fouquet-Chauprade \& Dutrévis). Stéréotypes sociaux, manque de formation pédagogique et de connaissance sur le contexte d'éducation prioritaire peuvent conduire les acteurs à limiter vis-à-vis des élèves leurs attentes, à ralentir les apprentissages, à valoriser les réussites ponctuelles au détriment de la construction des savoirs. Vis-à-vis des familles, ces mêmes représentations n'aident pas les parents à se construire comme partenaires de l'école. Une recherche sur les relations école-familles dans le REP genevois (Pelhate \& Rufin), même si l'on n'est pas exactement dans une pratique pédagogique, illustre cette explication : bien que très cadrées formellement, les rencontres enseignants-familles ne sont pas cadrées en termes de contenus et de visées ; les enseignants, non formés et non outillés à ce genre de pratiques, se sentent exposés et isolés, et finalement n'en tirent pas grand profit

5 Enseignement explicite: les élèves n'étant pas égaux dans leur capacité à faire le lien entre "réussir une tâche» et "comprendre, construire des savoirs", les apprentissages ne se réalisent pas si l'enseignant ne rend pas suffisamment explicites les objectifs, les méthodes d'apprentissages, les liens entre une activité et une notion en cours de construction, entre une notion déjà là et celle qui est travaillée, et s'il 
n'entraîne pas de façon systématique et explicite les élèves (Rochex). D'où la satisfaction de voir à partir de 2014 un travail d'instrumentation de la réflexion et de l'action des acteurs, un dispositif de formation spécifique, un équilibre entre les principes de bienveillance et d'exigence, et l'affirmation de la pédagogie explicite comme levier pédagogique. La pédagogie explicite a été retenue comme levier par les pays qui sont allés vers plus de prescriptions (États-Unis et Angleterre), comme elle a été mise en œuvre dans l'expérimentation menée dans le REP genevois (FouquetChauprade \& Dutrévis). En plus des deux contributions déjà citées (Bressoux \& Bianco ; Chesnais), la contribution de R. Goigoux et S. Cèbe pose également la question de l'intérêt d'un enseignement explicite ou par simple réitération. Mais on peut regretter qu'il ne soit rien dit des différences de conception et de mise en œuvre de la pédagogie explicite entre l'approche anglo-saxonne et québécoise d'une part et l'approche plus sociologique qui a présidé à la rédaction du Référentiel et a orienté les formations qui en ont découlé en France.

Intérêt d'un contre-exemple: le lecteur peut être dérouté par le compte rendu d'actions jugées inefficaces dans un collège (Nakhili \& Benhayoun), mais la contribution illustre en contre-exemple les trois explications avancées. Les acteurs manquent d'outillage théorique et pratique, l'analyse de la situation sur laquelle ils veulent agir souffre de confusion ou d'amalgame. Il s'agit certes d'un collège en $\mathrm{EP}$, mais les actions visent la prévention du décrochage et ce qui est dit du décrochage recoupe ce que l'on peut dire de tout élève en risque de décrochage. Les représentations des acteurs contrecarrent leurs actions : la question du rapport entre la dimension socio-affective et l'accès aux savoirs scolaires n'est pas examinée, les actions ne partent pas de l'analyse des difficultés des élèves mais du catalogue des ressources à disposition, le temps de concertation est sacrifié (même pour une co-intervention au prétexte de la confiance entre enseignants) au profit du temps passé auprès de l'élève comme si un rapport privilégié avec lui agissait " par nature ». L'élève concerné par une action n'en est pas informé, au prétexte de ne pas le stigmatiser, il n'en tire pas profit.

\section{Trois contributions éclairent le débat sur les différences de qualité d'enseignement en EP et hors EP}

7 Le chapitre rédigé par Soussi et Nidegger fait part des résultats d'une enquête par questionnaire sur leurs pratiques, menée auprès d'enseignants du REP genevois et d'enseignants hors REP. L'enquête vise à vérifier deux hypothèses, sur les différences de conditions d'enseignement et de climat scolaire en et hors REP, et sur l'adaptation des enseignants de REP à leurs élèves. Les auteurs concluent au peu de différenciation selon le contexte scolaire. La même formation des enseignants, leur relative homogénéité générationnelle, le fait d'avoir les mêmes programmes conduisent aux mêmes pratiques pédagogiques déclarées. La lecture des tableaux présentés par les auteurs est très éclairante, concernant par exemple le climat de la classe : on constate une convergence inattendue en et hors REP sur des items tels que « les élèves attendent avec impatience la fin de la leçon ", "veulent faire des progrès ", "s'intéressent à ce qu'on leur apprend », « sont attentifs pendant les leçons »... 
8 Les observations effectuées lors d'une vaste enquête sur les pratiques pédagogiques d'enseignement de la lecture et de l'écriture en $\mathrm{CP}$ mises en regard des résultats des élèves (Goigoux \& Cèbe) conduisent à une conclusion du même ordre : les enseignants en EP ne sont ni plus ni moins efficaces que leurs collègues hors EP. La méthodologie de l'enquête est précisément présentée (étude des effets des pratiques en "valeur ajoutée » sur les apprentissages en cours et non sur les acquis antérieurs; élaboration d'un modèle d'analyse tenant compte des performances initiales des élèves, de leurs caractéristiques sociodémographiques, des caractéristiques des classes et des enseignants; observation directe dans les classes par des chercheurs de treize laboratoires universitaires; observation du fonctionnement quotidien dans des classes ordinaires [variété des méthodes d'apprentissage, des matériels pédagogiques...] et exclusion des classes d'enseignants débutants ; sélection des zones géographiques et de contextes sociaux divers). Les observations visent à mettre en rapport les occasions d'apprendre proposées par les enseignants et les performances des élèves. Elles portent sur les tâches assignées aux élèves, non pas sur ce que fait chaque élève mais sur ce qui est attendu par le maître. Ainsi ont été reconstitués la durée et l'agencement temporel des tâches proposées (un tableau éclairant présente la liste des tâches et le «budgettemps" qui lui a été associé durant les trois semaines de test). L'enquête révèle la convergence du budget-temps moyen consacré au lire-écrire durant la période d'observation (trois semaines) : 7,19 heures hors EP et 7,29 heures en EP. À noter que l'étude ne porte que sur des maitres expérimentés en $\mathrm{CP}$, ce qui appelle une vigilance sur la gestion des ressources humaines si l'on veut garantir une efficacité comparable des enseignants en et hors EP.

9 La contribution d'A. Chesnais se fonde, elle, sur le lien entre le contexte d'enseignement, les pratiques enseignantes et leurs effets sur les apprentissages des élèves, s'inscrivant de façon critique dans la filiation des travaux de Rochex et Crinon (2011). L'auteure observe les mêmes séances consacrées à la notion d'angle en mathématiques et conduites par le même enseignant expérimenté dans deux classes de sixième de contexte différent (l'une en REP+, l'autre dans un collège "ordinaire » de banlieue) à une année d'écart. Elle s'intéresse aux activités des élèves - ce qu'ils font, disent et pensent pendant la réalisation de la tâche -, activités qui sont à l'origine des apprentissages et résultent en grande partie des pratiques enseignantes. La comparaison entre les séances sur les deux années porte sur la part et la nature des tâches effectivement données aux élèves, les apports de l'enseignant au-delà des tâches prévues dans le scénario, les aspects langagiers des activités des élèves et de l'enseignant, les moments d'exercices les plus riches qui contribuent le plus efficacement aux apprentissages. L'analyse porte sur les différences et les similitudes entre les deux classes et leurs effets différenciateurs possibles sur les apprentissages. Elle met au jour des phénomènes peu visibles dans les enquêtes déclaratives, par exemple dans un moment pédagogique où l'enseignant rend explicite un élément de savoir pour la classe " ordinaire » mais le laisse demeurer « invisible» dans la classe REP+ alors qu'il s'agit du même enseignant, persuadé de suivre le même scénario. C'est donc là où des différences entre EP et hors EP ne sont pas attendues et n'apparaissent pas à un regard ou une enquête trop lointains qu'il est intéressant de les analyser, et l'auteure le fait avec une précision et une profondeur qui font regretter que cette contribution intervienne si tard dans le recueil. 


\section{L'ensemble des contributions enrichit la réflexion sur quatre questions de recherche}

10 À quelles conditions cadrer l'acte pédagogique? L'exemple du REP genevois est clairement conclu: un dispositif, une modalité pédagogique sont efficaces si les enseignants "peuvent y adhérer ", donc ont été formés, sont volontaires pour la mise en œuvre, sont accompagnés durant celle-ci. La conclusion de l'enquête portant sur le dispositif PARLER (Bressoux \& Bianco) est tout aussi claire: posant la question de la transférabilité du dispositif, les auteurs soulignent que PARLER n'est pas une méthode mais un programme, un ensemble de recommandations, de principes d'enseignement (enseignement explicite et entraînement systématique), de modes d'organisation, fondé sur la recherche (evidence based) et mis en place par une co-construction entre chercheurs et enseignants. Pour prescrire, il faudrait donc renoncer au "prêt-àporter », l'exemple cité devant être une source d'inspiration et de motivation. Le rappel de l'échec des années 1990 en Angleterre (Fouquet-Chauprade \& Dutrévis) vient à point nommé confirmer ce double point de vue. La conclusion de G. Felouzis résume la réflexion sur la nécessité d'un cadrage, assis sur les acquis de la recherche, mais aussi de la formation et de l'accompagnement.

11 Y a-t-il ou non des différences d'enseignement entre EP et hors EP ? Le fait qu'on en constate là où on ne l'attend pas (Chesnais) et qu'on n'en constate pas là où on s'y attend, à Genève (Soussi \& Nidegger) comme en France (Goigoux \& Cèbe), invite à interroger un prisme possible, celui du niveau d'enseignement, et l'hypothèse selon laquelle on n'observerait pas ou peu de différences en début de scolarité, alors que ces différences iraient croissant au fur et à mesure qu'on monte dans le cursus ? Et, plus généralement, la question se pose de savoir si ce qu'on dit de l'éducation prioritaire en primaire vaut pour le collège.

12 Si oui, ces différences ont-elles un effet négatif? Si l'on observe des différences, ont-elles nécessairement un effet négatif sur les apprentissages? La lecture de l'ouvrage conduit à un constat non pas nuancé mais subtil : et si, au fond, l'adaptation de l'enseignant à ses élèves était le propre du professionnel de la pédagogie ? La différenciation est-elle plus négative qu'une absence de différenciation ? Faut-il s'adapter au risque de ralentir les apprentissages ? Faut-il ne pas s'adapter au risque d'empêcher les apprentissages? La différenciation continue bien d'apparaître comme une nécessité, mais ce que mettent en lumière plusieurs contributions, c'est la nécessité pour les enseignants d'avoir conscience de la différenciation qu'ils mettent ou non en œuvre et de ses effets induits, lesquels ne sont pas toujours ceux que l'on en attend.

Quelles observations en recherche pour quelles conclusions? Enfin, quelles méthodes de recherches pour quelles conclusions sur l'acte pédagogique? L'étude du REP genevois montre qu'il y a assez peu de différences dans les pratiques pédagogiques en et hors REP, et qu'elles n'apparaissent que lorsqu'on différencie dans le hors REP entre le "proche REP » et le "très favorisé ». Constat est fait également que les seules déclarations des enseignants ne suffisent pas à explorer les pratiques pédagogiques. C'est dans les classes, en observant, en filmant, en recueillant des traces de l'activité, pour étudier ensuite le détail des interactions entre enseignant et élèves, que les chercheurs pourront " affiner leur grain » et saisir les différences non dans les tâches demandées aux élèves mais aussi dans l'étayage que l'enseignant apporte pendant leur 
réalisation. La qualité des contributions d'A. Chesnais et de R. Goigoux et S. Cèbe plaide ainsi pour une observation au plus près de l'acte pédagogique.

14 L'intérêt de l'ouvrage est donc de présenter et d'analyser des méthodologies de recherche confrontées aux résultats des enquêtes menées. Plutôt qu'un panorama des pratiques pédagogiques il propose au lecteur une réflexion sur les spécificités de leur évaluation dans le contexte de l'éducation prioritaire.

\section{BIBLIOGRAPHIE}

CNESCO (2016). Comment l'école amplifie-t-elle les inégalités sociales et migratoires ? Paris : Ministère de l'Éducation nationale, de l'Enseignement supérieur et de la Recherche.

ROCHEX J.-Y. \& CRINON J. (dir.) (2011). La construction des inégalités scolaires. Au cour des pratiques et des dispositifs d'enseignement. Rennes : Presses universitaires de Rennes.

\section{AUTEURS}

\section{ANNE ARMAND}

Inspectrice générale de l'Éducation nationale honoraire 